\title{
Sternocleidomastoid Flap for Pedicled \\ Reconstruction in Head \& Neck Surgery- Revisiting the Anatomy and Technique
}

\section{Apurva Srivastava}

Sir Ganga Ram Hospital

Tarun Kumar ( $\nabla$ batra_tarun@hotmail.com )

Banaras Hindu University Institute of Medical Sciences https://orcid.org/0000-0002-9078-2821

\section{Shashi Kant Kumar}

Banaras Hindu University Institute of Medical Sciences

\section{R.C Shukla}

Banaras Hindu University Institute of Medical Sciences

\section{Esha Pai}

Banaras Hindu University Institute of Medical Sciences

\section{Manoj Pandey}

Banaras Hindu University Institute of Medical Sciences

\section{Research}

Keywords: Sternocleidomastoid flap, Superior thyroid artery based sternomastoid flap

Posted Date: October 1st, 2021

DOI: https://doi.org/10.21203/rs.3.rs-923907/v1

License: (1) This work is licensed under a Creative Commons Attribution 4.0 International License. Read Full License 


\section{Abstract}

Background:

Previous studies on sternocleidomastoid flaps, have defined the importance of preserving sternocleidomastoid (SCM) branch of superior thyroid artery (STA). This theory drew criticism, as this muscle is known to be a type II muscle, i.e., the muscle has one dominant pedicle (branches from the occipital artery at the superior pole) and smaller vascular pedicles entering the belly of muscle (branches from STA and thyrocervical trunk) at the middle and lower pole respectively. It was unlikely for the SCM branch of STA to supply the upper and lower thirds of the muscle. We undertook a cadaveric angiographic study to investigate distribution of STA supply to SCM muscle.

Methods:

It is a prospective study on 10 cadaveric SCM muscles along with ipsilateral STA which were evaluated with angiography using diatrizoate (urograffin) dye. Radiographic films were interpreted looking at the opacification of the muscle. Results were analyzed using frequency distribution and percentage.

Results:

Out of ten specimens, near complete opacification was observed in eight SCM muscle specimens. While one showed poor uptake in the lower third of the muscle, the other showed poor uptake in the upper third segment of muscle.

\section{Conclusion:}

Based on the above findings we propose to re-classify sternocleidomastoid flap as a type III flap as the STA branch also supplies the whole muscle along with previously described pedicle from occipital artery. However, this needs to be further corroborated intra-operatively using scanning laser doppler.

\section{Introduction:}

Microvascular surgery has become the backbone of complex reconstructions for head and neck resections but it does not reduce the importance of learning the techniques of pedicled flaps for a head and neck surgeon. When the situation demands or when means and expertise are not available, the surgeons have to fall back on pedicled flaps. One such flap is the pedicled sternocleidomastoid (SCM) myocutaneous flap.

Conventionally, the SCM flap has been described as a type II flap by Mathes and Nahai ${ }^{1}$, i.e. the muscle has one dominant pedicle (branches from the occipital artery at the superior pole) and smaller vascular pedicles entering the belly of muscle (branches from superior thyroid artery (STA) and thyrocervical trunk) at the middle and lower pole, respectively. 
This flap is usually not the preferred choice of most surgeons as total or partial flap loss has been reported in $10-30 \%$ of the cases ${ }^{2}$. However, flap loss may be minimized by modifying the technique to preserve the branch from superior thyroid artery (STA), as shown in our own series, resulting in flap loss

of $6.25 \%^{3}$. One of the criticisms of preserving the middle pedicle is that, it being a type II muscle, STA supplies only the middle segment of the muscle and does not contribute to blood supply of lower segment of the muscle. Hence, we re-visited the arterial anatomy of this flap with the help of cadaveric angiography.

\section{Methods:}

This is a prospective observational study where angiographic examination of the SCM muscle was performed on dissected specimens from cadavers to evaluate its blood supply. 10 specimens were dissected out from 5 cadavers, i.e. both right sided and left sided specimens. In the specimen, we included (Fig. 1):

1. The common carotid artery and its bifurcation into the external and internal carotid arteries.

2. The STA, along with its origin from the external carotid artery.

3. The SCM of the same side, keeping its pedicles from STA intact.

After placing an infant feeding tube into the common carotid artery, ligation was done at three sites:

1. At the proximal end of the common carotid artery.

2. At the distal end, both on the external and internal carotid arteries.

3. At the distal end of the STA, after the branch to SCM.

The lumen of the vessels was flushed with saline to remove all clots. A suitable contrast material, diatrizoate (Urograffin) was injected through the infant feeding tube from the proximal end. After 5 minutes, keeping it in anatomical position, a radiograph of the specimen was obtained. Radiographic films were interpreted looking at the opacification of the muscle. A radiolucent hue was considered as perfusion of the muscle segment.

Data was analyzed using frequency distribution and percentage. Institutional ethics committee approval was obtained before starting the study.

\section{Results:}

Out of ten specimens, we saw near complete opacification of eight SCM muscle specimens (Fig. 2). One specimen showed poor uptake in the lower third of the muscle, and another showed poor uptake in the upper third segment of muscle (Fig. 3 \&4) (Table 1- Opacification of muscle specimens).

All eight specimens wherein the whole muscle was opacified, showed uniform uptake in all the three segments of the muscle. 


\section{Discussion:}

Based on the above findings we propose that the SCM flap be re-classified as type III, instead of type II, as described previously in literature ${ }^{1}$. A type III flap has two dominant pedicles. According to the findings in this study, the STA is the second dominant pedicle supplying the SCM flap, other than its already established supply from the occipital artery (which serves as its first dominant pedicle). It is evident from the above findings that STA, not only augments the blood supply of the muscle (via occipital artery) but, is also capable of solely supplying the whole muscle in $80 \%$ of cases. STA supplied two-thirds of the muscle, including the lower third of the muscle, in $90 \%$ cases. In our previous series, we reported a flap loss of $6.25 \%$, by preserving the SCM branch of STA ${ }^{3}$, as opposed to the historical $10-30 \%$, reported in literature ${ }^{2}$. These clinical results are reinforced by our present angiographic study. In situations, where more length of the flap is required, the STA was ligated distal to the SCM branch ${ }^{3}$. Tiwari et $\mathrm{al}^{4}$ have described their series of 18 successful cases of SCM flaps, of which 3 flaps were done for temporal bone resection defects. In these 3 , they detached the muscle from the temporal bone, and none of the muscular branches of occipital artery were preserved. The flap survived solely on the SCM branch from STA, as well as collateral circulation from the branches of occipital and posterior auricular arteries supplying the overlying skin. Wei et al., in their series of 65 patients, emphasized on preserving the SCM branch of STA. They reported partial flap loss of $8 \%$ and did not report any total flap loss. Similarly, Khazaeni in their lone case ${ }^{4}$, reported sternocleidomastoid myocutaneous flap, for a full thickness cheek defect solely based on the SCM branch of STA, as the occipital branch was ligated during the lymph-node dissection in the same case. Jones et al. ${ }^{2}$ have very systematically described decrease in the complications of the SCM flap from about $60 \%$ in $1980 s^{5}$ to present rate of about $2 \%(2016)^{6}$. In their review, they attributed lower complication rates to: 1 ) suturing skin paddle to underlying muscle so that the perforators can be safeguarded from shearing, while handling the flap 2) to check the skin refill before suturing the flap to the defect 3) preservation of SCM branch of superior thyroid artery.

Hu et $\mathrm{al}^{7}$ in their series of 50 cadaveric muscle specimens, described a branch of STA, that runs down to the clavicle and supplies perforators to perfuse the lower third segment of the muscle. They also stressed on the importance STA in supplying the lower third of the muscle.

The relatively higher loss of the flap in various series may be due to poor underlying perforators to the overlying skin in which case, there is only a partial loss of the skin paddle while the underlying muscle remains healthy. This has been discussed in detail by Jabaley et al. ${ }^{8}$ where they studied the blood supply of sternocleidomastoid in 3 ways: cadaveric dissection, neck dissections and fluorescein dye testing, after the flaps were raised. They concluded that due to paucity of perforators from the muscle to skin or very narrow caliber capillaries traversing from muscle to overlying skin, there is a greater chance of skinpaddle loss that would eventually heal by re-epithelization with healthy underlying muscle. Another proposed reason for higher flap loss is disrupted venous drainage. As most of the discussions revolve around arterial anatomy of the muscle, venous drainage is often overlooked. SCM is drained by accompanying venous tributaries with each vascular pedicle and additionally by small tributaries to 
external and anterior jugular vein ${ }^{9}$. Every effort should be made to preserve maximum venous tributaries to prevent congestion of the flap.

The limitation of our study is a relatively small sample size, that may obviate anatomic variations in the blood supply and this requires larger numbers to be studied. Another drawback is the unaccounted entity

of "feeding-vessel" spasm, a phenomenon ${ }^{10-12}$ which may get aggravated by flap-handling. In real time surgery, there is always a chance of feeding vessels or perforators 'going into spasm' despite rich supply through intramuscular communications. As with all cadaveric studies, it was not possible to study this and it may very well be a cause of flap failure despite angiographic uptake in the lower third of the muscle.

\section{Conclusion:}

Based on the above findings, we propose to reclassify pedicled SCM flap as a type III flap. However, this should be further corroborated with intra-operative scanning laser doppler after occluding occipital artery. We also strongly recommend to preserve STA branch to the SCM along with the occipital artery for better perfusion of lower third of the flap, hence minimizing rates of flap loss and flap-related morbidity.

\section{Declarations}

Ethical Approval: Taken from the institutional ethics committee before starting the study- DEAN 2008$09 / 169$

Consent for Publication: Not Applicable

Availability of Data: On Request

Competing Interests: None

Funding: None

Authors Contribution: AS- Anatomical dissection and performance of procedure, TK- Preparation of manuscript and review of literature, SKP- Anatomical part of the study and concept, RCS- Radiological interpretation, MP- Overall concept of the study, manuscript preparation and editing.

Acknowledgements: The authors thank the anonymous individuals who selflessly donated their bodies for research and academic learning.

\section{References}

1. Mathes SJ, Nahai F. Classification of the vascular anatomy of muscles: Experimental and clinical correlation. Plast Reconstr Surg. 1981. doi:10.1097/00006534-198167020-00007. 
2. Jones LF, Farrar EM, Roberts DJH, Moor JW. Revisiting the sternocleidomastoid flap as a reconstructive option in head and neck surgery. J Laryngol Otol. 2019;133(9):742-6. doi:10.1017/S0022215119001592.

3. Kumar V, Gaud U, Shukla M, Pandey M. Sternocleidomastoid island flap preserving the branch from superior thyroid artery for the reconstruction following resection of oral cancer. Eur J Surg Oncol. 2009. doi:10.1016/j.ejso.2009.02.014.

4. Khazaeni K, Rajati M, Shahabi A, Mashhadi L. Use of a sternocleidomastoid myocutaneous flap based on the sternocleidomastoid branch of the superior thyroid artery to reconstruct extensive cheek defects. Aesthetic Plast Surg. 2013. doi:10.1007/s00266-013-0216-z.

5. Sasaki CT. The Sternocleidomastoid Myocutaneous Flap. Arch Otolaryngol. 1980;106(2):74-6. doi:10.1001/archotol.1980.00790260006003.

6. Ellabban MA. The Sternocleidomastoid Muscle Flap: A Versatile Local Method for Repair of External Penetrating Injuries of Hypopharyngeal-Cervical Esophageal Funnel. World J Surg. 2016. doi:10.1007/s00268-015-3306-z.

7. Hu KS, Song WC, Kim SH, et al. Branching patterns of the arterial branches supplying the middle vascular pedicle of the sternocleidomastoid muscle: A topographic anatomical study with surgical applications for the use of pedicles osteomuscular flaps. Surg Radiol Anat. 2006. doi:10.1007/s00276-005-0053-0.

8. Jabaley ME, Heckler FR, Wallace WH, Knott LH. Sternocleidomastoid regional flaps: A new look at an old concept. Br J Plast Surg. 1979. doi:10.1016/0007-1226(79)90007-9.

9. Bordoni B, Varacallo M. Anatomy, Head and Neck, Sternocleidomastoid Muscle. StatPearls Publishing; 2018. http://www.ncbi.nlm.nih.gov/pubmed/30422476. Accessed April 5, 2020.

10. Barron JN, Emmett AJJ. Subcutaneous pedicle flaps. Br J Plast Surg. 1965. doi:10.1016/S00071226(65)80005-4.

11. Bonde CT, Holstein-Rathlou NH, Elberg JJ. Blood flow autoregulation in pedicled flaps. J Plast Reconstr Aesthetic Surg. 2009. doi:10.1016/j.bjps.2008.07.039.

12. Arnold PB, Merritt W, Rodeheaver GT, Campbell CA, Morgan RF, Drake DB. Effects of perivascular botulinum toxin-A application on vascular smooth muscle and flap viability in the rat. Ann Plast Surg. 2009. doi:10.1097/SAP.0b013e3181903252.

\section{Tables}

Table 1- Opacification of muscle specimens 


\begin{tabular}{|ll|}
\hline Specimen Number & Opacification on dye study \\
\hline Subject 1R & Full Opacification \\
\hline Subject 1L & Full Opacification \\
\hline Subject 2R & Full Opacification \\
\hline Subject 2L & Full Opacification \\
\hline Subject 3R & Upper two thirds only \\
\hline Subject 3L & Full Opacification \\
\hline Subject 4R & Full Opacification \\
\hline Subject 4L & Full Opacification \\
\hline Subject 5R & Lower two thirds only \\
\hline Subject 5L & Full Opacification \\
\hline
\end{tabular}

\section{Figures}




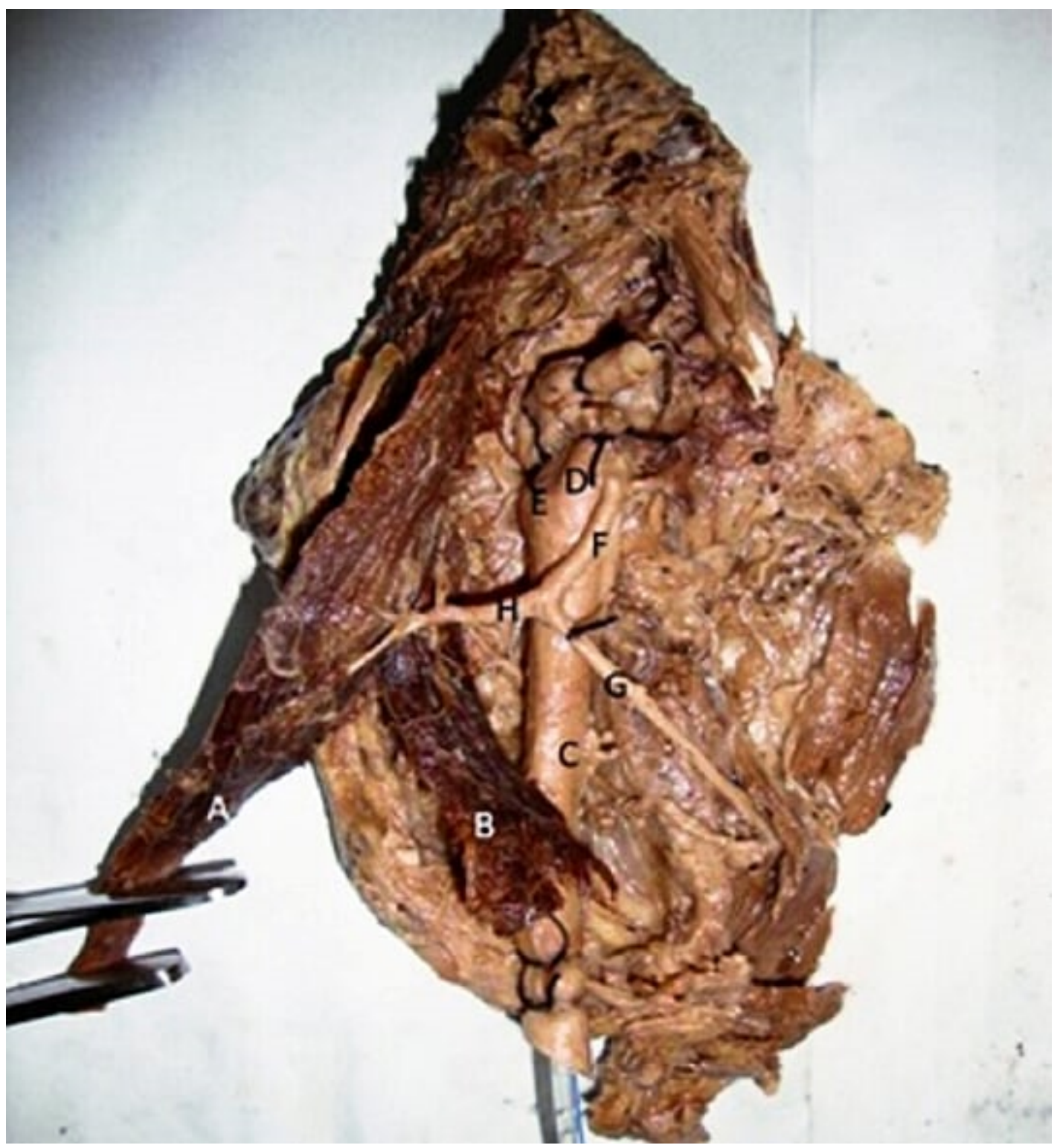

Figure 1

Specimen of SCM muscle with its blood supply. Infant feeding tube can be seen in common carotid artery. Internal carotid artery and external carotid artery distal to STA is ligated. STA distal to SCM branch is also ligated. (A: Clavicular head of sternocleidomastoid, B: Sternal head of sternocleidomastoid, C: Common carotid artery, D: External carotid artery, E: Internal carotid artery, F: Superior thyroid artery, G: Thyroid branch of superior thyroid artery, $\mathrm{H}$ : Sternomastoid branch of superior thyroid artery) 


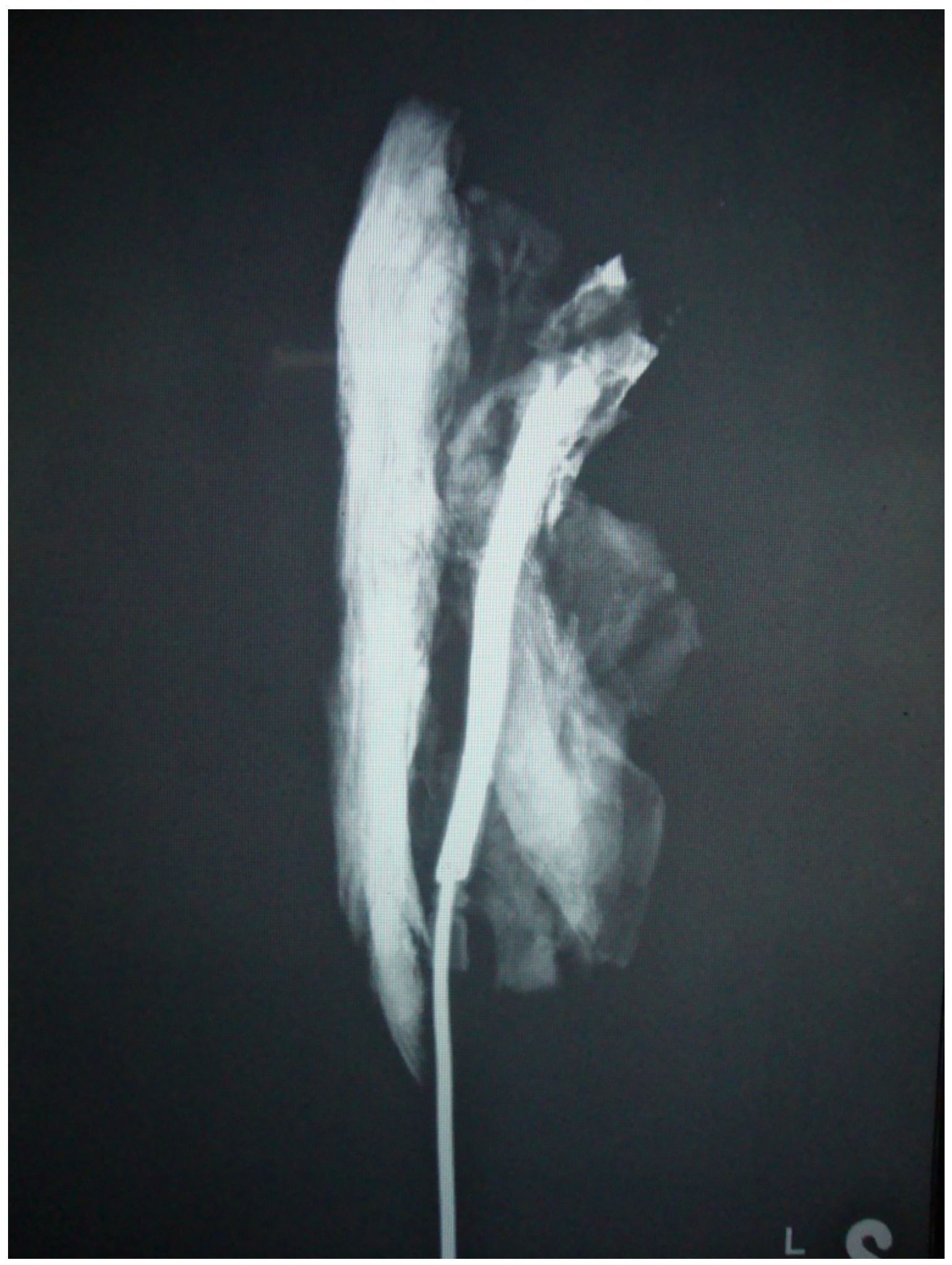

Figure 2

Angiographic film showing complete opacification of the muscle. 


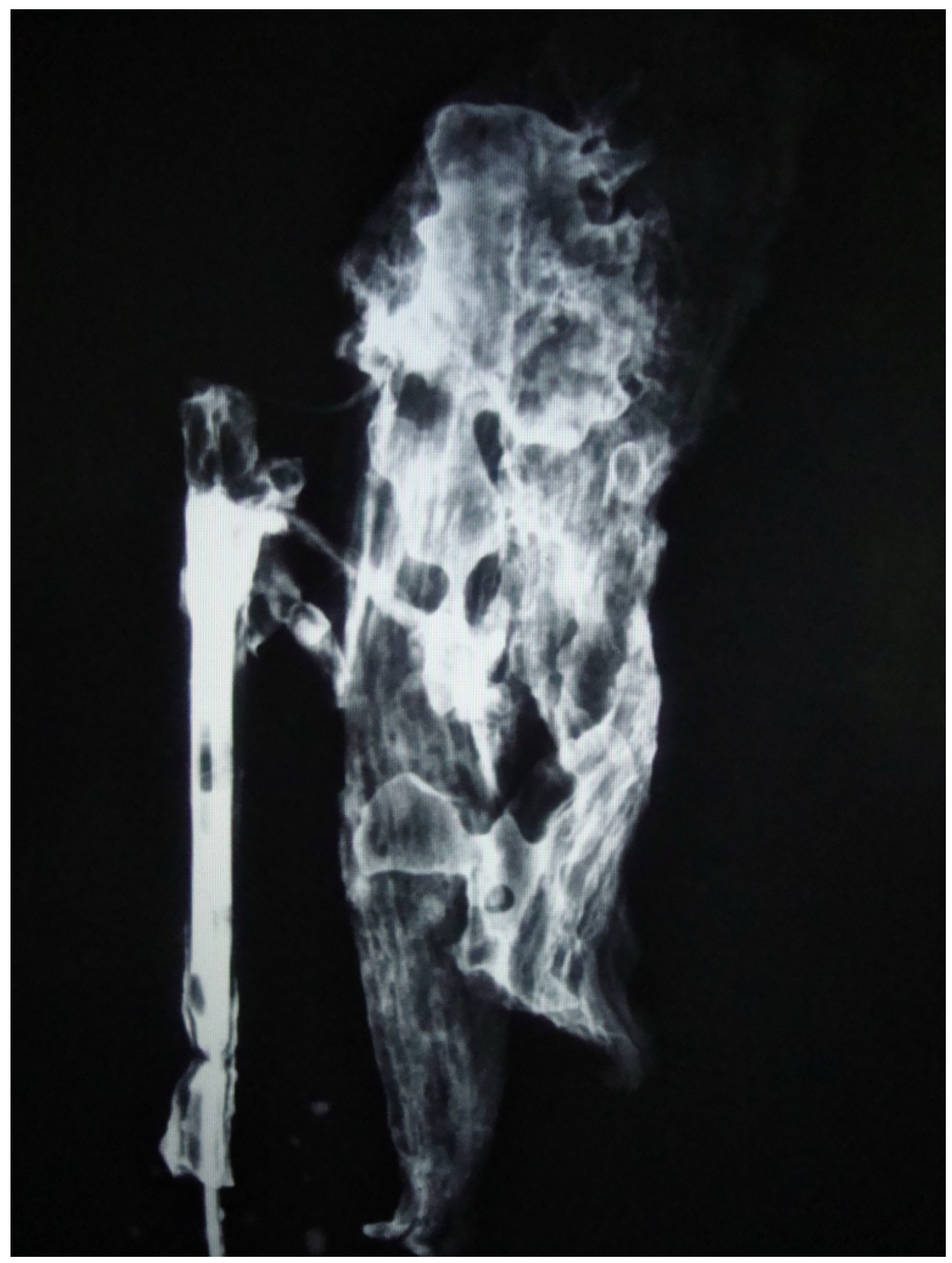

Figure 3

Angiographic film showing un-opacified lower third. 


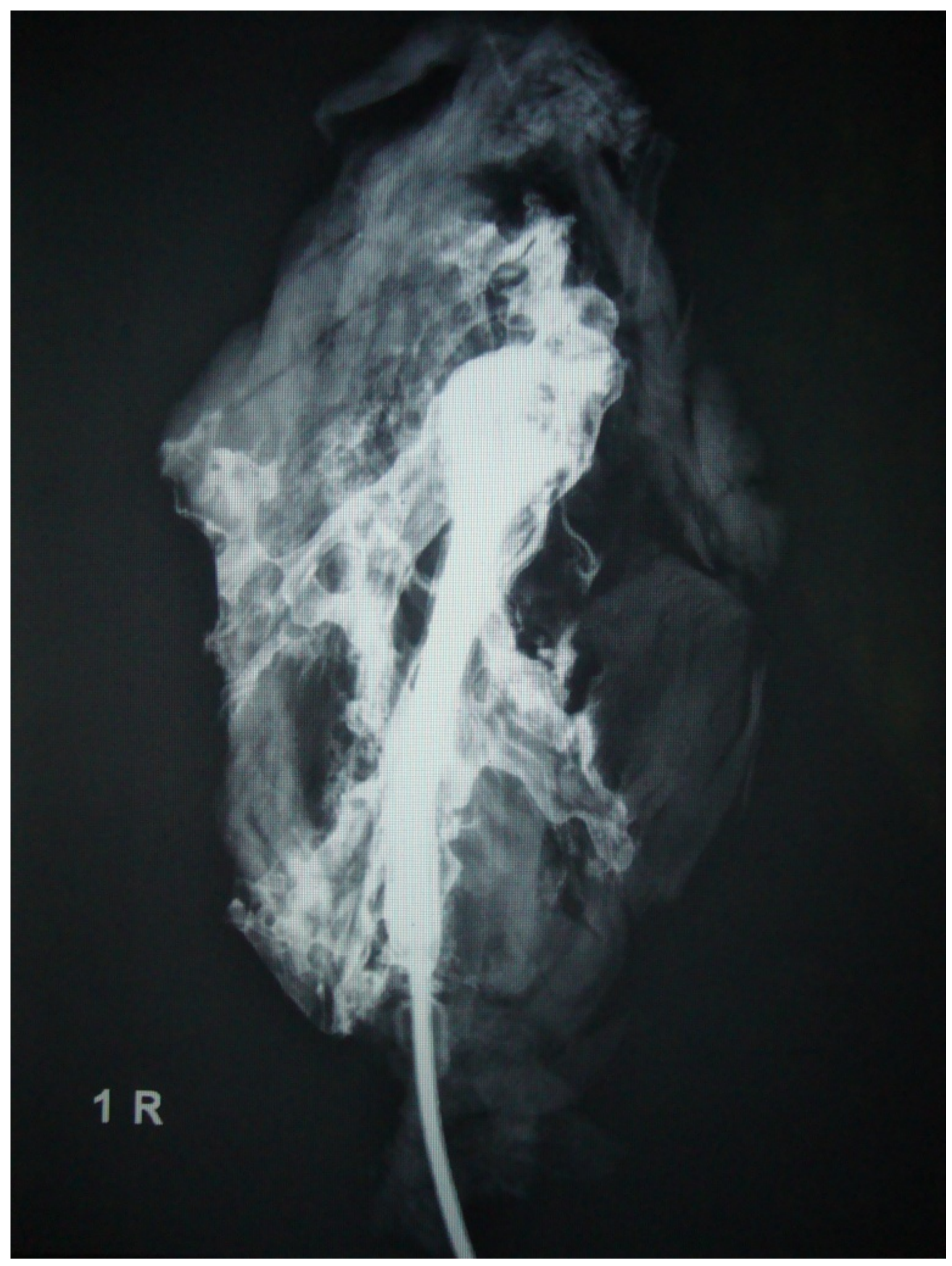

Figure 4

Angiographic film showing un-opacified upper third. 